\title{
Mobile and Trapped Triplet States in Single Crystals of Charge Transfer Complexes
}

\author{
H. Möhwald ${ }^{1}$ and E. Sackmann \\ Max-Planck-Institut für biophysikalische Chemie, Karl-Friedrich-Bonhoeffer-Institut, \\ Abt. Spektroskopie, Göttingen, Germany
}

(Z. Naturforsch. 29 a, 1216-1228 [1974] ; received April 27, 1974)

\begin{abstract}
Homogeneously doped crystals of charge transfer(CT-) complexes were grown by incorporating aromatic guest donors in host CT-crystals. The host crystals contained 1,2,4,5-tetracyanobenzene (TCNB) as acceptor and deuterated aromatic electron donors. By using such doped crystals CT. complexes in a well defined configuration may be studied. The triplet states of the guest complexes were used as ESR spectroscopic probes in order to determine the molecular arrangement in the host lattice. The zero-field-splitting (ZFS) parameters, $D$ and $E$, of the triplet energy traps were determined and the degree of electron delocalization in the triplet state was calculated from these values. In some cases a very strong guest host interaction (multicomplex formation) was established. A method for the determination of CT-triplet energies is described (accuracy $200 \mathrm{~cm}^{-1}$ ). The phosphorescence spectrum of the anthracene-TCNB complex was obtained from the delayed emis sion spectra of different anthracene doped CT-crystals. The vibronic structure is identical to that of anthracene, while the 0.0 -band of the complex is blue shifted by $600 \mathrm{~cm}^{-1}$.

It is shown that the undoped anthracene-TCNB crystal exhibits P-type delayed fluorescence and that the triplet exciton diffusion in this crystal is nearly temperature independent. In the undoped biphenyl-TCNB crystal E-type delayed fluorescence originating from the thermal depopulation of the mobile triplet excitons is established. The remarkable differences of the two types of triplet excitons are interpreted in terms of the different polarity in the triplet states of the two CT. crystals.
\end{abstract}

\section{Introduction}

Aromatic electron donors and acceptors tend to form 1:1 mixed crystals where the two types of molecules are alternatively stacked in linear arrays with the molecular planes being parallel. As the distance between the molecular planes is rather small $(3.4 \AA)$ the intermolecular forces in a direction parallel to the stack axis are very large compared to the interactions between the stacks. The stacking is favoured by Coulomb forces caused by a slight electron delocalization from the donor to the acceptor in the ground state of the complex. Accordingly, crystals of charge transfer (CT.) complexes may be considered as one-dimensional electronic systems. Such crystals are very useful as model systems to study the interaction between closely packed charge transfer complexes in fixed relative positions. The interest in linear arrays of planar and well polarizable molecules has been mainly stimulated by the search for superconducting organic solids ${ }^{2,3}$.

In this work we studied crystals of CT-complexes containing 1,2,4,5-tetracyanobenzene (TCNB) as electron acceptor and different aromatic hydrocarbons as electron donors. The lowest excited singlet

Reprint requests to Helmuth Möhwald, IBM Research Laboratory, San José, California 95114. states of complexes with TCNB are characterized by a strong charge transfer from the donor to the acceptor. In contrast the degree of electron delocalization in the lowest excited triplet states varies over a wide range for different donors.

It has been demonstrated recently by ESR spectroscopy that the triplet states of the CT-crystals biphenyl-TCNB and anthracene-TCNB form highly mobile excitons ${ }^{4,5}$. Depending on the change in the degree of charge delocalization upon excitation the motion of such charge transfer triplet excitons is more or less thermally activated. In the present work we found that the crystals biphenyl-TCNB and anthracene-TCNB exhibit strong delayed fluorescence as a consequence of the high exciton mobility. The results of the emission spectroscopic and the ESR spectroscopic studies are compared.

The main part of the present work deals with ESR and optical studies of CT-crystals containing the acceptor TCNB that are doped with different guest donors. In many cases the lowest excited triplet states of the guest complexes form traps in the host crystal. This enables one to study

(a) CT-complexes in a fixed configuration

(b) the interaction and the energy transfer between different CT-complexes. In addition, the lowest excited guest triplet states are used as structure sensi-
This work has been digitalized and published in 2013 by Verlag Zeitschrift für Naturforschung in cooperation with the Max Planck Society for the Advancement of Science under a Creative Commons Attribution-NoDerivs 3.0 Germany License. 
tive probes to determine the molecular arrangement in the host crystal. In the mixed crystals the phosphorescence spectrum of the guest complexes could also be observed in favourable cases.

\section{Experimental}

TCNB was prepared in this laboratory by Dr. W. Kühnle. It was purified by recrystallization from ethanol and by subsequent sublimation. The donors naphthalene- $\mathrm{d}_{8}$, biphenyl- $\mathrm{d}_{10}$ and anthracene- $\mathrm{d}_{10}$ (Merck, Sharp \& Dohme) were repeatedly zonerefined. Anthracene- $d_{10}$ that was recrystallized from ethanol and vacuum sublimed before zone-refining was a gift from Dr. B. Nickel. The donors durene- $d_{14}$, pyrene- $d_{10}$ and phenanthrene- $d_{10}$ (Merck, Sharp \& Dohme) were used without further purification. In order to test the influence of possible impurities we also prepared crystals with undeuterated donors that were zone-refined several times. The ZFS parameters of the crystals with purified and unpurified donors were identical.

The crystals were grown from spectroscopically pure acetone solutions containing equimolar quantities of TCNB and one donor. For the preparation of the doped crystals a small amount of the guest donor was added to these solutions. The crystals formed needles and had the typical dimensions $4 \times 1 \times 0.5 \mathrm{~mm}^{3}$.

The guest concentration was kept as low as possible (between $1 \%$ and $1 \%$ ) in order to obtain homogeneously doped host crystals. For the crystals containing anthracene and pyrene as guests the guest concentrations could be determined from the absorption spectra obtained after dissolving the crystal in an appropriate solvent. In all other cases the concentrations were estimated from the relative concentrations contained in the solutions from which the mixed crystals were grown.

The triplet ESR spectra were taken with a Varian E 12 spectrometer. The crystals were mounted on cylindrical quartz rods in such a way that the magnetic field could be rotated in the different crystallographic planes. Irradiation was achieved with a $100 \mathrm{~W}$ high pressure $\mathrm{Hg}$ lamp (Osram HBO $100 \mathrm{~W} /$ $2)$. Cut-off filters were used in order to excite only the lowest excited charge transfer singlet states of the crystal. For measurements at temperatures below $80 \mathrm{~K}$ the spectrometer was equipped with a helium flow cryostate (Leybold Heraeus).

The emission spectra were recorded with a device described in detail by B. Nickel and U. Nickel ${ }^{6}$. Excitation of the crystals was effected by one line $(476 \mathrm{~nm})$ of a krypton ion laser (Coherent Radia- tion $52 \mathrm{~B} \mathrm{KUV).} \mathrm{Frequency} \mathrm{and} \mathrm{duration} \mathrm{of} \mathrm{the}$ excitation was varied by choppers. Another chopper in the emission light path was used to measure the spectra at different times after the excitation. The emission was detected with a red sensitive photomultiplier (EMI 9658-20). The decay curves were stored in a multichannel analyzer (Ortec 6220). The accuracy of the decay time measurements was better than $0.1 \mathrm{~ms}$.

\section{ESR Investigations of Doped CT-Crystals}

\section{A) Guest Triplet States as Structure Sensitive} Probes. Molecular Arrangement in the CT-Crystal Naphthalene-TCNB: According to the X-ray analysis of Kumakara et al. ${ }^{7}$ the crystal naphthalene (naph)TCNB is monoclinic with two complexes per until cell $(a=9.39 \AA, b=12.66 \AA, c=6.87 \AA, \beta=107.2 \AA)$. The molecular planes are oriented perpendicular to the stack axis $c$. The long axis $x$ of the acceptor lies parallel to the axis $a^{\prime}$, perpendicular to $b$ and $c$. The donor may assume two different orientations, where the long axis forms an angle of $+18^{\circ}$ or $-18^{\circ}$ with the $a^{\prime}$ axis.

Figure 1 shows the room temperature triplet ESR spectra of an anthracene (anth) doped naph-TCNB crystal for different orientations of the magnetic field in the $a^{\prime} b$ plane. For each orientation two gaussian absorption lines appear which have a line width of about 10 Gauss for anth- $h_{10}$ and a line width of about 5 Gauss for anth- $\mathrm{d}_{10}$ (cf. insert of Figure 1).

Anth-TCNB forms low lying triplet traps in the host crystal. Accordingly the observed ESR spectra are expected to be due to the anth-TCNB guests. By measuring the ESR spectra for magnetic field orientations that were slightly tilted against the $c$ axis we found that this crystallographic axis is a principal axis of the zero-field-splitting (ZFS) tensor $^{8}$. The orientations $\alpha=0^{\circ}$ and $\alpha=90^{\circ}$ in Fig. 1 that yield the extrema of the resonance fields then define the other two principal axes. Tentatively assigning the $x, y$ and $z$ axis to the $a^{\prime}, b$ and $c$ axis, respectively, one obtains the ZFS parameters $D= \pm 0.0669 \mathrm{~cm}^{-1}$, $E=\mp 0.00751 \mathrm{~cm}^{-1}, \quad D^{\mathrm{m}}=\left(D^{2}+3 E^{2}\right)^{1 / 2}=0.068$ $\mathrm{cm}^{-1}$. These values are nearly identical with the corresponding values of both the pure anthracene and the isolated anth-TCNB complex (see Table 1). Since anth and anth-TCNB have identical ZFS tensors ${ }^{5}$, it 

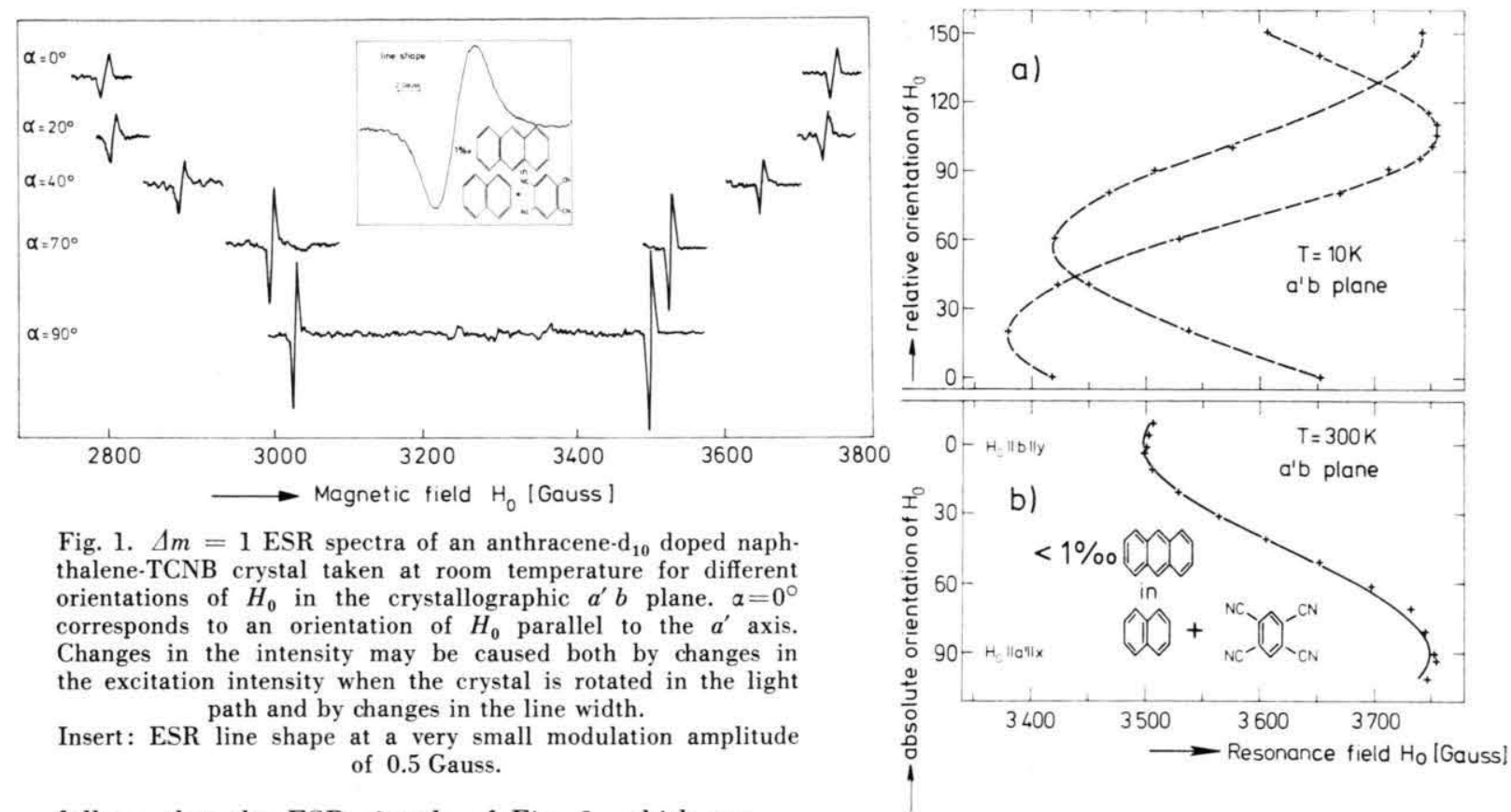

Fig. 1. $\Delta m=1$ ESR spectra of an anthracene- $d_{10}$ doped naphthalene-TCNB crystal taken at room temperature for different orientations of $H_{0}$ in the crystallographic $a^{\prime} b$ plane. $a=0^{\circ}$ corresponds to an orientation of $H_{0}$ parallel to the $a^{\prime}$ axis. Changes in the intensity may be caused both by changes in the excitation intensity when the crystal is rotated in the light path and by changes in the line width.

Insert: ESR line shape at a very small modulation amplitude of 0.5 Gauss.

follows that the ESR signals of Fig. 1, which can now be assigned to the anth-TCNB complex, yield information on the orientation of the anthracene guest donor in the crystal matrix. It follows from Fig. 1 and from Fig. $2 \mathrm{~b}$ that at room temperature all anthracene molecules are translationally equiv. alent. It is reasonable to assume that the donor anthracene orients parallel to the host donors. If this is true one should expect from the above mentioned X-ray results two differently oriented guest traps. In contrast to this expectation one observes only one pair of lines for each orientation at $300 \mathrm{~K}$. Figure $2 \mathrm{a}$ shows that at very low temperatures $(\sim 10 \mathrm{~K})$ one observes indeed two translationally inequivalent guest traps yielding two pairs of ESR lines. The extrema appear at orientations that differ by about $35 \pm 5^{\circ}$. For the orientation " $c \| H_{0}$ " the two pairs of lines coincide. Accordingly at low temperatures the anthracene molecules may assume two different orientations where the $x$ axes form an angle of $35^{\circ}$, while the planes of all molecules are parallel.

This supports the results of the X-ray analysis concerning the orientation of the naphthalene donors and furthermore confirms our previous assumption that the anthracene guest orients in the same way as the host donors. At higher temperatures $(300 \mathrm{~K})$ the anthracene molecules obviously quickly change between the two possible orientations so that only one

Fig. 2. Angular dependence of the high field line of Fig. 1 as measured at room temperature and at a very low temperature. The crosses denote measured points.

a) $T=10 \mathrm{~K}$. At this temperature it was difficult to rotate the magnetic field exactly in a crystallographic plane. The deviations in the minimum and the maximum resonance fields in Figs. $2 \mathrm{a}$ and $2 \mathrm{~b}$ are most probably due to this uncertainty. The microwave frequency $v$ was $9199 \mathrm{Mc}$.

b) $T=300 \mathrm{~K}$. The solid curve has been calculated with the ZFS parameters given in Table I. $v=$ $9239.5 \mathrm{Mc}$.

average orientation is observed in the ESR spectra *.

From the above discussion one should expect that the ZFS parameters obtained at $300 \mathrm{~K}$ and at $10 \mathrm{~K}$ are different. However, within our experimental accuracy this difference could not be detected, since the canonical resonance fields corresponding to the $x$ and $y$ axis differ only by about $250 \mathrm{Gauß}$.

Molecular arrangement in the CT-crystals biphenyl-TCNB, durene-TCNB and phenanthrene$T C N B$ : Using the method described above we also determined the ZFS parameters and the orientations of anth in durene-TCNB, biphenyl-TCNB and phenanthrene-TCNB. The results are summarized in Table I.

* It is expected that the freezing in of the donor librational motion is accompanied by a phase transition. In the anthTCNB crystal such a phase transition has recently been established from the temperature dependence of the ESR line width of the triplet excitons ${ }^{5}$. 
Table I. Molecular arrangement in some CT-crystals and ZSF parameters of anthracene and anth-TCNB in different environment. $s$ is the stack axis, $c$ is the normal to the molecular planes of the donors and $N$ is the number of translationally inequivalent donors per unit cell at room temperature.

\begin{tabular}{|c|c|c|c|c|c|c|}
\hline System & $\begin{array}{l}D\left[\mathrm{~cm}^{-1}\right] \\
( \pm 0.002)\end{array}$ & $\begin{array}{l}E\left[\mathrm{~cm}^{-1}\right] \\
( \pm 0.0005)\end{array}$ & $\begin{array}{l}D_{\mathrm{m}}^{\mathrm{m}}\left[\mathrm{cm}^{-1}\right] \\
( \pm 0.0025)\end{array}$ & $N$ & \multicolumn{2}{|c|}{$\begin{array}{l}\Varangle(z, s) s[\AA] \\
\left( \pm 5^{\circ}\right)\end{array}$} \\
\hline Anth in biphenyl-TCNB & \pm 0.063 & $\mp 0.008$ & 0.064 & 1 & 0 & 6.8 \\
\hline Anth in naph-TCNB & \pm 0.067 & $\mp 0.008$ & 0.068 & 1 & $0 \mathrm{~d}$ & $6.87 \mathrm{~d}$ \\
\hline Anth in durene-TCNB & \pm 0.068 & $\mp 0.008$ & 0.070 & 2 & 20 & 7.4 \\
\hline Anth in phenanth.-TCNB & \pm 0.067 & $\mp 0.008$ & 0.069 & 1 & 25 & \\
\hline Anth-TCNB (excitons) & $\pm 0.067 \mathrm{a}$ & $\mp 0.009 \mathrm{a}$ & $0.068 \mathrm{a}$ & 1 & 22 e & $7.4 \mathrm{e}$ \\
\hline Anth-TCNB (liq. cryst.) & & & $0.072 \mathrm{~b}$ & & & \\
\hline Anthracene (X-traps) & $\pm 0.069 \mathrm{e}$ & $\mp 0.008 \mathrm{c}$ & $0.070^{\mathrm{c}}$ & & & \\
\hline
\end{tabular}

a ZFS parameters from Ref. 5 .

b ZFS parameter from Ref. 27.

c ZFS parameters from Ref. 28.

d values from Ref. 7 .

e values from Ref. 29 .
By X-ray experiments based on the rotating crystal method we determined the dimensions of the stack axes $s$ for biphenyl-TCNB $(s=b=6.8 \AA)$ and durene-TCNB $(s=7.4 \AA)$. The dimensions of the stack axes $s$ and the angles between $s$ and the normals to the molecular planes are summarized in Table I. It is seen that even in cases where $s$ is not normal to the molecular planes the interplanar distance varies only between $3.4 \AA$ and $3.5 \AA$.

Table I further shows that the ZFS parameters of the anth-TCNB traps are almost equal in the different host crystals studied.

\section{B) ZFS Parameters of CT-Crystals Doped with other Donors}

The anth-TCNB complex is distinguished by a very low triplet energy and thus forms traps with an exceptionally high depth. At temperatures below $250 \mathrm{~K}$ we could also observe ESR spectra of CTcrystals doped with several other donors forming less deep guest traps. We applied the method des- cribed above in order to determine the orientation of the principal axes of the ZFS tensor in the host crystal as well as the ZFS parameters $D, E$ and $D^{\mathrm{m}}$. It has been found that all guest donors considered orient in the same way as the host donors in accordance with the results obtained for the anthracene guest. The ZFS parameters of the different guest host systems are summarized in Table II. For comparison the ZFS parameters of isolated CT-complexes and donors in a liquid crystalline solvent are given.

At low temperatures $(10 \mathrm{~K})$ we could also observe the localized triplet excitons in undoped crystals of some of the CT-complexes studied in the present paper. The ZFS parameters of these self-traps are also given in Table II.

\section{C) Evaluation of the "Degree of Electron Delocalization" $c_{1}^{2}$ from ZFS Parameters}

A comparison of the ZFS parameters of an isolated complex with the values obtained when the same complex is incorporated in a host crystal shows

Table II. ZFS parameters $\left(D, E, D^{\mathrm{m}}\right)$, degrees of electron delocalization $\left(c_{1}{ }^{2}\right)$, changes in $c_{1}{ }^{2}$ induced by guest host interaction $\left(\delta c_{1}^{2}\right)$, and activation energies $(\Delta E)$ for different guest host systems.

\begin{tabular}{|c|c|c|c|c|c|c|}
\hline & $\begin{array}{l}D\left[\mathrm{~cm}^{-1}\right] \\
( \pm 0.002)\end{array}$ & $\begin{array}{l}E\left[\mathrm{~cm}^{-1}\right] \\
( \pm 0.001)\end{array}$ & $\begin{array}{l}D_{\mathrm{m}}^{\mathrm{m}}\left[\mathrm{cm}^{-1}\right] \\
( \pm 0.003)\end{array}$ & $c_{1}{ }^{2}$ & $\delta c_{1}^{2}$ & $\Delta E\left[\mathrm{~cm}^{-1}\right]$ \\
\hline pyrene in naph-TCNB & \pm 0.041 & $\mp 0.0024$ & 0.058 & 0.36 & 0.27 & 2190 \\
\hline pyrene in durene-TCNB & \pm 0.057 & $\mp 0.0038$ & 0.086 & 0.055 & -0.035 & 1400 \\
\hline pyrene in biphenyl-TCNB & \pm 0.057 & $\mp 0.0030$ & 0.077 & 0.155 & 0.065 & 750 \\
\hline pyrene-TCNB in liq. cryst. a & \pm 0.062 & $\mp 0.0032$ & 0.083 & 0.09 & 0.0 & \\
\hline pyrene-TCNB (crystal) & & & 0.081 & 0.11 & 0.02 & \\
\hline pyrene in liquid crystal a & \pm 0.069 & $\mp 0.0035$ & 0.091 & & & \\
\hline phenanthrene in naph-TCNB & \pm 0.036 & $\mp 0.0022$ & 0.052 & 0.59 & 0.01 & 770 \\
\hline phenanthrene in durene-TCNB & \pm 0.026 & $\mp 0.0017$ & 0.040 & 0.70 & 0.12 & 1270 \\
\hline phenanthrene in biphenyl-TCNB & \pm 0.0225 & $\mp 0.0017$ & 0.0375 & 0.72 & 0.14 & 375 \\
\hline phen. TCNB in liquid crystals a & \pm 0.039 & $( \pm) 0.0020$ & 0.054 & 0.58 & 0.0 & \\
\hline phenanthrene in liquid crystals b & \pm 0.104 & $\mp 0.00465$ & 0.132 & & & \\
\hline biphenyl in naph-TCNB & \pm 0.034 & $\mp 0.0014$ & 0.040 & 0.70 & 0.16 & 1100 \\
\hline biphenyl-TCNB (excitons) & \pm 0.053 & $\mp 0.0017$ & 0.060 & 0.54 & 0.0 & \\
\hline durene in naph-TCNB & \pm 0.025 & $\mp 0.0012$ & 0.032 & 0.76 & 0.03 & smaller than \\
\hline durene-TCNB (crystal) & \pm 0.034 & $\mp 0.0006$ & 0.035 & 0.73 & 0.0 & $100 \mathrm{~cm}^{-1}$ \\
\hline
\end{tabular}

a ZFS parameters taken from Ref. 27.

b ZFS parameters taken from Ref. 11 . 
that in many cases $D^{\mathrm{m}}$ decreases dramatically by incorporating the CT-complex in a host crystal [for example pyrene-TCNB in naph-TCNB (Table II)]. It is well known that a decrease in $D^{\mathrm{m}}$ is caused by an increase in the average distance between the two unpaired electrons. A change in the average distance of these two electrons may be expressed in terms of a change in the so called degree of electron delocalization, $Q . Q$ is equal to the square of the coefficient $c_{1}$ describing the contribution of the ionic resonance structure ${ }^{3} \psi$ '(ionic) to the wave function of the triplet trap:

$$
\begin{aligned}
{ }^{3} \psi(\text { trap })=c_{1}{ }^{3} \psi(\text { ionic }) & +c_{2}{ }^{3} \psi\left(A D_{\mathrm{g}}{ }^{*}\right) \\
& +c_{3}{ }^{3} \psi\left(A^{*} D_{\mathrm{g}}\right) .
\end{aligned}
$$

The functions ${ }^{3} \psi\left(A^{*} D_{\mathrm{g}}\right)$ and ${ }^{3} \psi\left(A D_{\mathrm{g}}{ }^{*}\right)$ in Eq. (1) take account for contributions of locally excited triplet states of the acceptor and the guest donor, respectively.

Admixtures of locally excited host donor states may be neglected. Such an approximation is justified as the overlap of locally excited triplet states of the host donor with the ionic state ${ }^{3} \psi$ (ionic) of an adjacent guest complex is very small ${ }^{9}$. Simple perturbation theory suggests that only contributions of the lowest locally excited triplet state need to be considered. Accordingly in the case of pyrene-TCNB and phenanthrene-TCNB as guests we consider only the lowest excited donor triplet state $\left(c_{3}=0\right)$, while in the case of the biphenyl-TCNB and the dureneTCNB guests only the lowest excited acceptor triplet state is important $\left(c_{2}=0\right)$. Neglecting off-diagonal terms ${ }^{10,11}$ Eq. (11) leads to

$$
c_{1}{ }^{2}=\frac{D\left({ }^{3} D_{\mathrm{g}}{ }^{*}\right)-D(\text { trap })}{D\left({ }^{3} D_{\mathrm{g}}{ }^{*}\right)-D(\text { ionic })}=\frac{E\left({ }^{3} D_{\mathrm{g}}{ }^{*}\right)-E(\text { trap })}{E\left({ }^{3} D_{\mathrm{g}}{ }^{*}\right)-E(\text { ionic })}
$$

for the case $c_{3}=0$.

Identical equations where $D\left({ }^{3} D_{g}{ }^{*}\right)$ and $E\left({ }^{3} D_{g}{ }^{*}\right)$ are replaced by $D\left({ }^{3} A^{*}\right)$ and $E\left({ }^{3} A^{*}\right)$ are obtained for the case $c_{2}=0 . D\left({ }^{3} D_{\mathrm{g}}{ }^{*}\right)$ and $E\left({ }^{3} D_{\mathrm{g}}{ }^{*}\right)$ are the ZFS parameters of the guest donors and $D$ (trap) and $E$ (trap) are the ZFS parameters of the traps.

In principle $c^{2}{ }_{1}$ may be calculated from Eq. (2), if the ZFS parameters $D$ (ionic) and $E$ (ionic) are known. Unfortunately, experimental values of these parameters are not available. Moreover, for TCNB only the value of $D^{\mathrm{m}}=\left(D^{2}+3 E^{2}\right)^{1 / 2}$ is known. Accordingly, one must try to evaluate $c_{1}{ }^{2}$ from the $D^{\mathrm{m}}$. value, an approach that has been made by Hayashi et al. ${ }^{10}$. These authors derived a relation similar to Eq. (2), where $D$ and $E$ are replaced by $D^{\mathrm{m}}$. However, Krebs ${ }^{12}$ has shown that these equations may lead to very large errors in calculating $c_{1}{ }^{2}$. He also showed that the relations of Hayashi et al. ${ }^{10}$ are only justified

(a) if $D$ is large compared to $E$ and

(b) if $D\left({ }^{3} A^{*}\right), D\left({ }^{3} D^{*}\right)$ and $D$ (ionic) do not have different signs.

Beens et al. ${ }^{11}$ and Hayashi et al. ${ }^{10}$ showed that for an isolated complex in its equilibrium configuration $D^{\mathrm{m}}$ (ionic) $=D^{\mathrm{m}}\left(A^{-} D^{+}\right) \approx 0.02 \mathrm{~cm}^{-1}$. The observed $D^{\mathrm{m}}$-values of the localized triplet states studied in the present work range from $D^{\mathrm{m}} \approx 0.09 \mathrm{~cm}^{-1}$ to $D^{\mathrm{m}} \approx 0.13 \mathrm{~cm}^{-1}$. Since in CT-crystals the electron delocalization is often more pronounced (see below), one would expect $D^{\mathrm{m}}$ (ionic) $\leqq 0.02 \mathrm{~cm}^{-1}$. Accordingly the contribution of $D^{\mathrm{m}}$ (ionic) in the denominator of the equation given by Hayashi et al. ${ }^{10}$ may be neglected. In this approximation these relations become more justified. For the systems studied in the present work $c_{1}{ }^{2}$ may thus be calculated from

$$
\begin{aligned}
& c_{1}{ }^{2}=\frac{D^{\mathrm{m}}\left({ }^{3} D_{\mathrm{g}}{ }^{*}\right)-D^{\mathrm{m}}(\operatorname{trap})}{D^{\mathrm{m}}\left({ }^{3} D_{\mathrm{g}}{ }^{*}\right)} \\
& \quad \text { or } c_{1}{ }^{2}=\frac{D^{\mathrm{m}}\left({ }^{3} A^{*}\right)-D^{\mathrm{m}}(\operatorname{trap})}{D^{\mathrm{m}}\left({ }^{3} A^{*}\right)}
\end{aligned}
$$

to an accuracy of $20 \%$.

In isolated complexes only the electron delocalization between two molecules ( $A$ and $D$ ) has to be considered [ ${ }^{3} \psi^{\prime}($ ionic $\left.) \equiv{ }^{3} \psi\left(A^{-} D^{+}\right)\right]$and $c_{1}{ }^{2}$ is called the charge transfer character ${ }^{10.11}$. In a crystal the wave function ${ }^{3} \psi$ (ionic) is not well defined, since the two triplet electrons may be distributed over more than two molecules. In the above approximation for the calculation of $c_{1}{ }^{2}$, where $D^{\mathrm{m}}$ (ionic) is neglected, the exact form of the wave function ${ }^{3} \psi$ (ionic) need not be known.

The degrees of electron delocalization $c_{1}{ }^{2}$ calculated from Eq. (3) are summarized in Table II. In this table the differences $\delta c_{1}{ }^{2}$ in the degree of electron delocalization of the isolated complex and of the complex embedded in a CT-crystal are also given.

The systematic error introduced by application of Eq. (3) shifts the values of $c_{1}{ }^{2}$ for a given complex in different matrices in the same direction. Accordingly the differences $\delta c_{1}{ }^{2}$ are much more accurate than the absolute values of the degree of electron delocalization. 


\section{D) Determination of Triplet Energies from Trap Depths}

Figure 3 shows the temperature dependence of the ESR-intensity $I$ of various guest host systems * . For every system there exists at least one temperature region where the temperature dependence of $I$ is described by an equation $I=I_{0} \exp \{-\Delta E / k T\}$. In some cases two such regions may be distinguished (see Fig. 3 b, curves $2,3,4$ ). In Table II are given the activation energies $\Delta E$ obtained from the straight lines in the regions with the strongest temperature dependence.
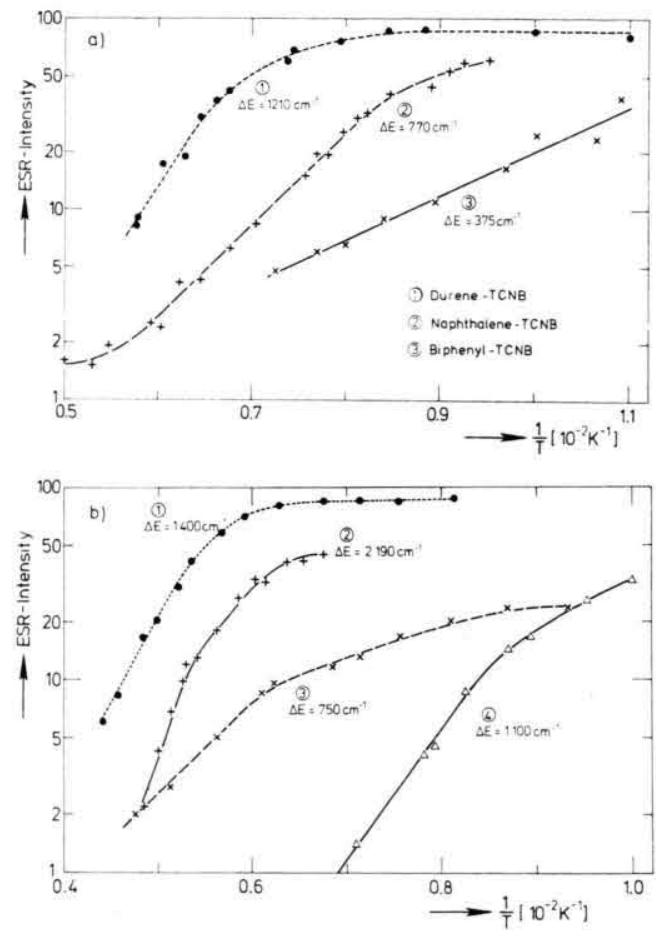

Fig. 3. Temperature dependence of the ESR intensity for different guest host systems. For each system the Arrhenius plot clearly exhibits at least one temperature region characterized by a straight line. The activation energies, $\Delta E$, given in the Fig. were obtained from the straight lines with the largest slope.

a) (1) phenanthrene in durene-TCNB, (2) phenanthrene in naph-TCNB, (3) phenanthrene in biph-TCNB.

b) (1) pyrene in durene-TCNB, (2) pyrene in naph-TCNB, (3) pyrene in biph-TCNB, (4) biphenyl in naph-TCNB.

The ESR spectra of the very deep anth-TCNB traps can be observed up to $400 \mathrm{~K}$ without any appreciable change in intensity and lifetime. This supports our previous assumption ${ }^{13}$ that in doped crystals the temperature dependence of the ESR intensity is mainly determined by the thermal de- population of the guest triplet state via the host triplet exciton band. Therefore the activation energies $\Delta E$ of Table II are equal to the energy difference between the lowest excited guest and host triplet state ${ }^{14}$.

Provided the guest host interaction is negligible the energy of CT triplet states may be determined to an accuracy of about $200 \mathrm{~cm}^{-1}$. As an example consider the crystal phenanthrene in naph-TCNB. Since the ZFS parameter $D^{\mathrm{m}}$ of the guest complex and of the isolated complex phenanthrene-TCNB in a liquid crystalline solution are about equal (cf. Table II), the guest host interaction is negligible. Accordingly, it follows from the values of $\Delta E$ given in Table II that the triplet energy of phenanthreneTCNB is nearly $800 \mathrm{~cm}^{-1}$ lower than that of naphTCNB.

This information can hardly be obtained from the phosphorescence spectra of isolated complexes (a) since the spectra are very broad (half width $2000 \mathrm{~cm}^{-1}-3000 \mathrm{~cm}^{-1}$ for polar triplet states) and (b) since the ground state of CT-complexes may be considerably lowered by resonance interaction with excited CT singlet states.

Since the ZFS parameters of different host crystals containing the same guest vary to a large extent, the question arises whether the observed ESR signal must be assigned to the guest or to the host complex.

From optical data one can predict that the triplet energies of anth-TCNB and pyrene-TCNB are much lower than the triplet energies of the hosts used in this work. Only for these guest host systems can the observed ESR spectra be assigned unambigously to the guest complex. For all other guest host systems it is not possible to decide from optical spectra alone whether the guest or the host CT triplet state is lower in energy.

In these cases an assignment was achieved by comparing the ESR spectra obtained for a host crystal A doped with a guest complex B with the spectra observed for a system where B was the host and $\mathrm{A}$ was the guest. As an example consider the system biphenyl in naph-TCNB. Obviously the observed ZFS parameters of this system differ drastically from the values obtained for the isolated biphenyl-TCNB complex (cf. Table II). Nevertheless we ascribe the observed ESR signal of the doped

\footnotetext{
* The ZFS parameters and the ESR line widths are tem. perature independent.
} 
crystal to the lowest excited triplet state of biphenylTCNB from the following reasons: The crystal naph in biphenyl-TCNB exhibits no ESR signal of a localized triplet state at temperatures above $100 \mathrm{~K}$. Only the mobile triplet excitons of the host crystals are observed in this temperature range. On the other hand the crystal biphenyl in naph-TCNB exhibits an ESR signal of a very deep trap. Therefore the observed ESR signal must be assigned to the biphenylTCNB triplet state which is much lower in energy than the naph-TCNB triplet state.

Using the same simple procedure the ESR spectra obtained for the crystals doped with phenanthreneTCNB had to be assigned to the guest complex.

In the same way it has been found that the dureneTCNB triplet state is by only about $100 \mathrm{~cm}^{-1}$ lower in energy than the naph-TCNB triplet state.

\section{E) Lowering of Triplet States and Determination of Charge Transfer Triplet Energies}

The degrees of electron delocalization $c_{1}{ }^{2}$ and the triplet trap depths may be compared in Table II. As an example consider the host crystals naph-TCNB and durene-TCNB containing pyrene-TCNB as a guest. As shown above the triplet energies of both host crystals are about equal. Therefore in naphTCNB the triplet energy of pyrene-TCNB is by $800 \mathrm{~cm}^{-1}$ lower than in durene-TCNB. Table II also shows that the $c_{1}{ }^{2}$-value of pyrene-TCNB in dureneTCNB is considerably smaller than the value of $c_{1}{ }^{2}$ obtained when this complex is incorporated in naphTCNB. In this example a lowering in the triplet energy is accompanied by an increase in the degree of electron delocalization. This is also valid for all the other guest host systems summarized in Table II.

According to simple perturbation theory the lowering $\delta(\Delta E)$ in the guest triplet energy is proportional to the increase $\delta c_{1}{ }^{2}$ in the contribution of the ionic structure ${ }^{3} \psi$,(ionic) to the wave function $3_{y}{ }^{\prime}$ (trap) [cf. Equation (1) ].

$$
\delta(\Delta E)=K \delta c_{1}{ }^{2} .
$$

Assuming further that the proportionality constant $K$ does not depend considerably on the nature of both the guest and the host complex the change in the trap depth caused by the guest host interaction may be determined from the change in the ZFS parameters. Therefore the above mentioned method (cf. III. D) for the determination of CT triplet energies may be extended to systems with strong guest host interaction provided $K$ is known. A value of the constant $K$ and the triplet energies ${ }^{3} E^{\circ}$ of the isolated (unperturbed) CT-complexes were obtained as follows: From the values of $\delta c_{1}{ }^{2}$ and $\Delta E$ for the two crystals pyrene in naph-TCNB and pyrene in durene-TCNB one obtains $K=3000 \mathrm{~cm}^{-1}$ and ${ }^{3} E^{\circ}$ (naph-TCNB) $=1400 \mathrm{~cm}^{-1}$ by defining ${ }^{3} E^{\circ}$ (pyrene$\mathrm{TCNB})=0$. This estimate is possible, since as shown above the triplet energies of the two host crystals naph-TCNB and durene-TCNB are equal. From the measurements of pyrene in biphenyl-TCNB one obtains the value of ${ }^{3} E^{\circ}$ (biphenyl-TCNB) (heavy line in Figure 4). Using the values of $A E$ and $\delta c_{1}{ }^{2}$ obtained for different host crystals doped with phenanthrene the triplet energy of the unperturbed phenanthrene-TCNB complex could be determined in three independent ways. The corresponding values given in Fig. 4, which differ by less than $200 \mathrm{~cm}^{-1}$ demonstrate the accuracy and the consistency of the method.

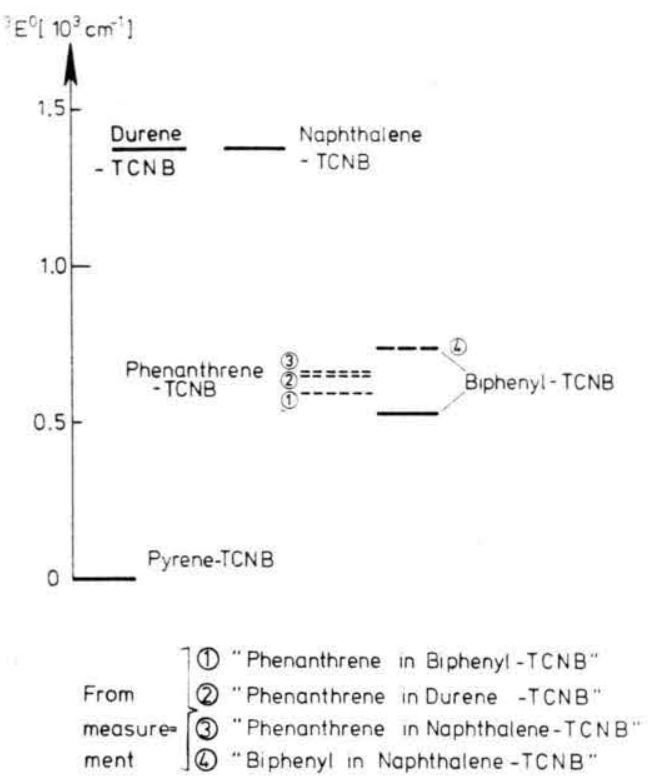

Fig. 4. CT triplet energies of isolated CT-complexes, determined by the procedure described in Chapter III.E. The pyrene-TCNB triplet energy has been arbitrarily defined as the energy zero point.

\section{F) Extended Electron Delocalization}

A basic assumption in the above discussion and in deriving Eq. (3) was that the observed decrease in $D^{\mathrm{m}}$ is caused primarily by an increase in the degree of electron delocalization. This implies that changes in the ZFS parameters $D$ (ionic) and $E$ (ionic) due to 
distortions of the guest complex configuration in the crystal matrix may be neglected. Indeed, quantum chemical calculations of $D\left(\mathrm{~A}^{-} \mathrm{D}^{+}\right)$and $E\left(\mathrm{~A}^{-} \mathrm{D}^{+}\right)$ for isolated CT-complexes by de Jong ${ }^{15}$ have shown that changes in both the distance and the mutual orientation of the donor and the acceptor affect the ZFS parameters of the ionic state ${ }^{3} \psi$ (ionic) only slightly.

The question arises whether the guest host interaction increases the degree of charge transfer within one donor acceptor pair or whether it causes an electron delocalization over more than two molecules. We exclude the first possibility for the following reason. A decrease in the ZFS parameter is accompanied by a decrease in the triplet energy of the trap. Thus the configuration with the lowest triplet energy of an isolated complex is expected to be that with the highest charge transfer character. Therefore we conclude that the triplet electrons of the trap in the CT-crystal are not confined to the guest complex, but that an electron delocalization over more than two molecules takes place. Such an extended charge delocalization may be considered as a multicomplex formed in the lowest excited triplet state. The existence of multicomplex formation in crystals of CT. complexes has been postulated several years ago by Briegleb ${ }^{16}$. In solution triple complex formation in the lowest excited singlet states has been reported previously by Beens and Weller ${ }^{17}$.

\section{Optical Studies}

\section{A) Delayed Emission from Doped CT-Crystals}

The doped CT-crystals exhibit strong delayed emission and were thus also studied by optical spectroscopy. However, in many cases the emission bands of the guest and host complex were very broad and structureless and overlapped strongly. Structured emission spectra could only be observed from the mixed crystals containing either anthracene or pyrene as guest donors ${ }^{1}$. In the following the emission spectra of mixed crystals with the guest donor anthracene will be discussed in detail.

As an example spectrum (4) in Fig. 5 shows the delayed emission of a naph-TCNB crystal containing anthracene as a guest. It has been found that all anth doped mixed crystals exhibit delayed emission maxima at $655 \mathrm{~nm}$ and at $722 \mathrm{~nm}$. Curve (1) in Fig. 5 shows that the prompt fluorescence of the pure anth-

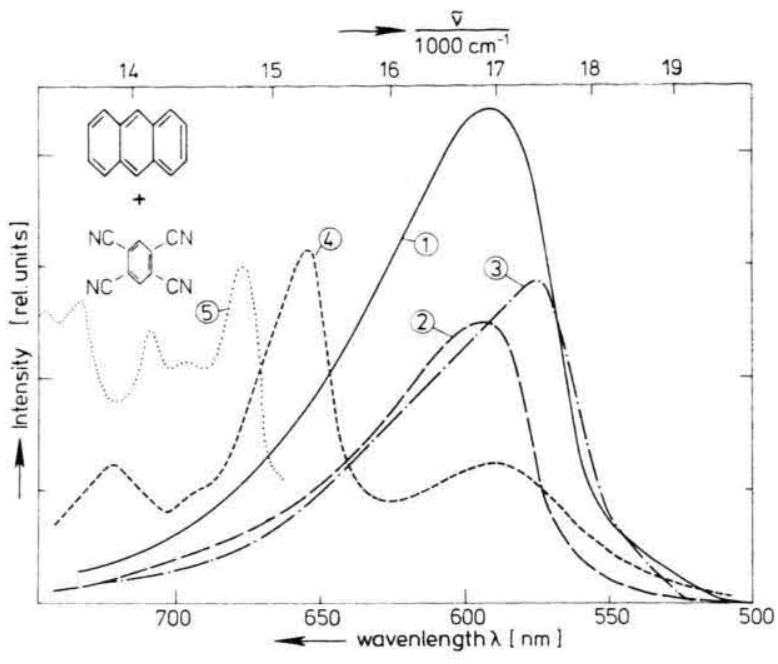

Fig. 5. Uncorrected emission spectra of crystals containing anthracene. (Slit width $3 \mathrm{~nm}$.) (1) prompt fluorescence of the crystal anth-TCNB at $300 \mathrm{~K}$. (2) and (3) delayed emission of the crystal anth-TCNB at $300 \mathrm{~K}$ and at $77 \mathrm{~K}$, respectively. (4) delayed emission of the anthracene doped naph-TCNB crystal at $300 \mathrm{~K}$. The maxima at $655 \mathrm{~nm}$ and at $722 \mathrm{~nm}$ correspond to the phosphorescence maxima of the anth-TCNB complex (cf. Chapter IV. A). (5) Corrected phosphorescence spectrum of the anth crystal at room temperature according to Reference 30 .

TCNB crystal is broad and structureless with a maximum at $590 \mathrm{~nm}$. Therefore the bands at $655 \mathrm{~nm}$ and at $722 \mathrm{~nm}$ of curve (4) in Fig. 5 have to be attributed to the anth-TCNB phosphorescence. The delayed emission spectra of all anth doped CT-crystals exhibit a broad band with a maximum between $560 \mathrm{~nm}$ and $590 \mathrm{~nm}^{1}$. Obviously these bands are caused by a superposition of the delayed fluorescence (DF) of anth-TCNB with the host phosphorescence.

A comparison of curves (4) and (5) in Fig. 5 shows that the vibronic structure of the anth-TCNB phosphorescence spectrum reflects the same progressions as the anth phosphorescence. However, the bands of the anth-TCNB complex are considerably broadened. The similarity in the phosphorescence spectra of anth and anth-TCNB is understandable on the basis of the ESR results showing that the guest triplet state is almost a pure anth triplet state. According to Fig. 5 the $\mathrm{O}-\mathrm{O}$ band of the anthTCNB phosphorescence is blue shifted by about $600 \mathrm{~cm}^{-1}$ with respect to the $\mathrm{O}-\mathrm{O}$ band of the anth phosphorescence. The ESR spectroscopic studies also showed that the absolute triplet energies of anth and anth-TCNB are equal. Therefore the ground state of the complex must have been lowered by about 
$600 \mathrm{~cm}^{-1}$ as a consequence of its resonance interaction with excited CT singlet states. Similar results and observations concerning the blue shift of the phosphorescence of the complex anth-pyromellitic dianhydrid at $1.6 \mathrm{~K}$ were also obtained by Haarer and $\mathrm{Karl}^{18}$.

It should be pointed out that the incorporation of CT-complexes in suitable CT-crystals may be a most useful method for studying the phosphorescence spectra of CT-complexes. It is much more difficult, for instance, to obtain the phosphorescence spectrum of anth-TCNB by other methods: The delayed emission spectrum of the undoped crystal anth-TCNB is composed solely of DF at temperatures above $100 \mathrm{~K}$ (cf. Chapter IV.B). The complex phosphorescence of a glassy solution containing the components is strongly overlapped by the donor phosphorescence. The overlap of the phosphorescence spectrum of a CT-complex with the spectrum of the corresponding donor is a well known drawback in optical studies of CT triplet states in solution ${ }^{16,19}$.

\section{B) Delayed Emission of the Undoped anth-TCNB Crystal}

Comparison of the spectra (1), (2) in Fig. 5 shows that the anth-TCNB crystal exhibits only fluorescence in the delayed emission spectrum. Even at $77 \mathrm{~K}$ no complex phosphorescence of the crystal is observed. The broad delayed emission band undergoes a blue shift upon lowering the temperature (cf. Figure 5). As in the case of anth-pyromellitic dianhydrid ${ }^{18}$ one may ascribe this to a freezing in of some excited state configurations.

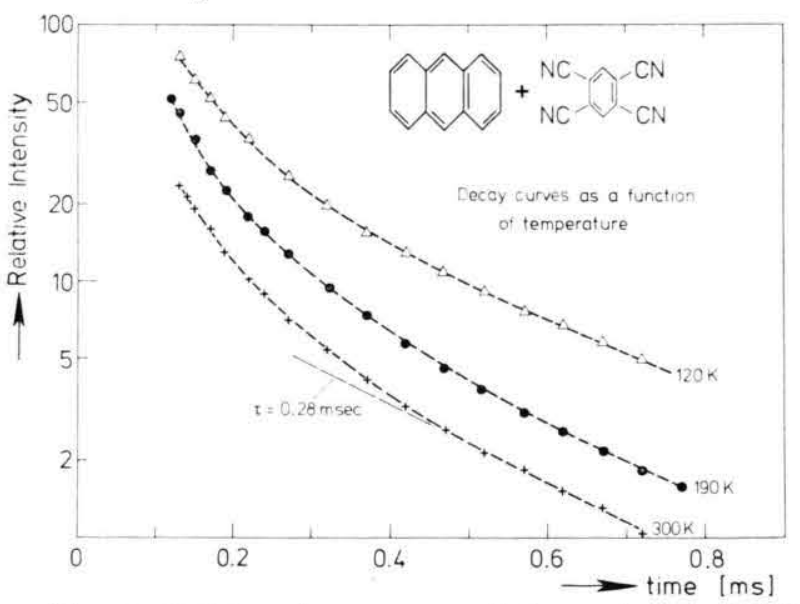

Fig. 6. Emission decay curves of the anthracene-TCNB crystal as a function of temperatures. Emission wavelength $600 \mathrm{~nm}$. The absolute intensities of the curves for different temperatures are not comparable.
To answer the question whether the DF of the crystal is of $E$-type or of $P$-type we studied the decay of the emission as a function of emission wavelength, excitation intensity and temperature. The results of these experiments (described in ref. 1) may be summarized as follows (cf. Figure 6) :

(a) The shape of the decay curves depends on the excitation intensity.

(b) The decay is only exponent:al at long times after excitation (Fig. 6) or at very low excitation intensities.

(c) The absolute emission intensities, $I_{1}$ and $I_{2}$, as measured at the same time after excitation for two different excitation intensities, $A_{1}$ and $A_{2}$, obey an equation of the form:

$$
I_{1} / I_{2}=\left(A_{1} / A_{2}\right)^{n} \text { with } 1.2<n<2 .
$$

(d) The decay curves do not depend on the emission wavelength showing that only one excited state emits light.

According to Parker ${ }^{20}$ observations (a), (b) and (c) demonstrate that the DF is generated predominantly by the biphotonic triplet-triplet annihilation $(P$-type). This author calculated decay curves as a function of a parameter R. This parameter denotes the ratio of the number of triplet states undergoing triplet-triplet annihilation to the number of triplet states decaying by first-order processes. Comparison of the calculated and the experimental curves given in Fig. 6 yields a value of $R=5 \pm 2$. Thus about $80 \%$ of the triplet states are deexcited by triplettriplet annihilation.

This result clearly shows that the lowest excited triplet state in the anth-TCNB crystal forms highly mobile triplet excitons in complete accordance with a previous ESR spectroscopic study. The triplet annihilation rate, which is proportional to $R$, is then determined by the rate of encounters of the triplet excitons. Accordingly $R$ is proportional to the triplet exciton diffusion coefficient $D_{\mathrm{d}}$. The analysis of the decay curves of Fig. 6 in terms of the parameter $R$ shows that $R$ decreases by less than a factor of two if the temperature is decreased from $300 \mathrm{~K}$ to $120 \mathrm{~K}$. Hence the triplet exciton diffusion coefficient varies only slightly with temperature. The ESR measurements reported previously ${ }^{5}$ showed that $D_{i 1}$ is constant in the temperature intervals between $4 \mathrm{~K}$ and $100 \mathrm{~K}$ and between $210 \mathrm{~K}$ and $350 \mathrm{~K}$, respectively. A phase transition caused by a reorientation 
of the molecules was observed at $T_{\mathrm{t}}=205 \mathrm{~K}$. Therefore the absolute values of the diffusion coefficient, obtained from the ESR line narrowing, in the two regions above and below $T_{\mathrm{t}}$ could not be compared. However, the delayed fluorescence spectroscopy shows that the diffusion coefficient does not change appreciably with the temperature even in the region near the transition temperature $T_{\mathrm{t}}$ (curve for $190 \mathrm{~K}$ in Figure 6).

From the decay time $\tau_{\mathrm{DF}}$ of the DF at low excitation intensities one also obtains the lifetime $\tau_{0}$ of the triplet excitons in the absence of triplet-triplet annihilation. From Fig. 6 one obtains $\tau_{0}=2 \tau_{\mathrm{DF}} \approx$ $0.5 \mathrm{~ms}$.

\section{C) Delayed Emission of the Undoped Biphenyl-TCNB Crystal}

Curve (5) in Fig. 7 a shows that the fluorescence spectrum of the crystal biphenyl-TCNB is very broad and structureless. Due to efficient reabsorption the position of the maximum at $520 \mathrm{~nm}$ can only be located to an accuracy of $20 \mathrm{~nm}$. Within this uncertainty the maximum of spectrum (5) coincides with the fluorescence maximum of the biph-TCNB complex in solution (maximum at $510 \mathrm{~nm}$ ).

The delayed emission spectrum of the crystal taken at $77 \mathrm{~K}$ [curve (4) in Fig. 7 a)] agrees well with the phosphorescence spectrum of the biphTCNB complex in a glassy solution (maximum at $550 \mathrm{~nm}$, lifetime $1.0 \mathrm{sec}$ ). Thus spectrum (4) in Fig. 7 a must be attributed to the phosphorescence of biph-TCNB self-traps in the crystal. At $340 \mathrm{~K}$ a broad band with a maximum at $580 \mathrm{~nm}$ appears in the delayed spectra (cf. Figure $7 \mathrm{~b}$ ). We attribute this band with a rather long lifetime of the emitting species $\left(30 \mathrm{~ms}^{1}\right)$ to the phosphorescence of biphTCNB traps. This requires that the phosphorescence spectrum undergoes a red shift with increasing temperature. The argument for explaining the blue shift of the anth-TCNB fluorescence upon lowering the temperature may also be applied to the biphTCNB phosphorescence. Accordingly one expects that by increasing the temperature from $77 \mathrm{~K}$ to $340 \mathrm{~K}$ the phosphorescence maximum shifts from $555 \mathrm{~nm}$ to $580 \mathrm{~nm}$.

Hence the band with a maximum at $490 \mathrm{~nm} \pm$ $10 \mathrm{~nm}$ appearing at shorter times after excitation (Fig. $7 \mathrm{~b}$ ) must be ascribed to delayed fluorescence. Curves (1), (2) and (3) in Fig. 7 a demonstrate
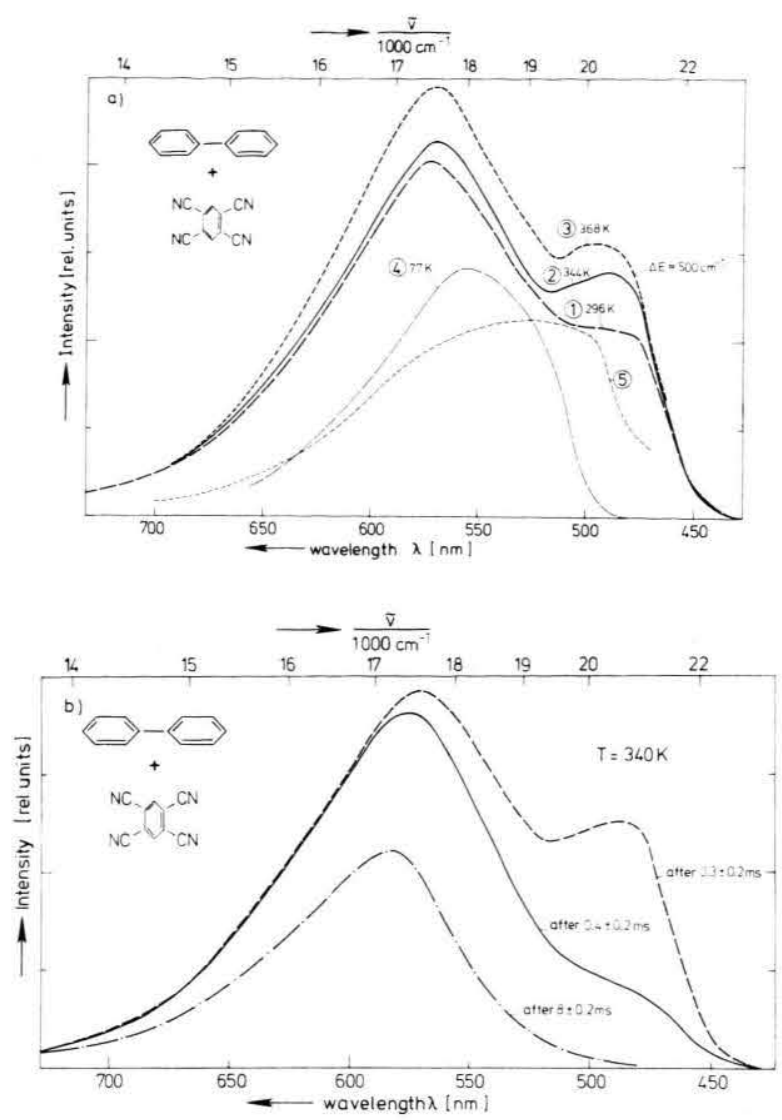

Fig. 7. Emission spectra (uncorrected) of the biphenyl. TCNB crystal (slit width $3 \mathrm{~nm}$ ).

a) (1), (2) and (3) delayed emission at different temperatures integrated in the time interval between $0.1 \mathrm{~ms}$ and $0.5 \mathrm{~ms}$ after excitation. The intensities of the spectra are comparable. (4) delayed emission at $77 \mathrm{~K}$ recorded $18 \mathrm{~ms}$ after excitation. (5) prompt emission at $300 \mathrm{~K}$.

b) Time dependent emission spectra taken at $340 \mathrm{~K}$. The emission was integrated in a $0.4 \mathrm{~ms}$ time interval while starting at different times $(0.1 \mathrm{~ms}, 0.2 \mathrm{~ms}$ and $7.8 \mathrm{~ms})$ after excitation.

that this band increases strongly with increasing temperature. This finding also supports the conclusion that the $490 \mathrm{~nm}$ band in Fig. $7 \mathrm{~b}$ originates from DF, since any trap emission would decrease with increasing temperature. From the temperature dependence of the emission intensity at $490 \mathrm{~nm}$ one obtains an activation energy of $\Delta E_{D \mathrm{~F}}=500 \pm$ $200 \mathrm{~cm}^{-1}$ for the formation of DF.

In order to find out which mechanism leads to the DF we also studied the decay of the emission as a function of excitation intensity and wavelength (at $300 \mathrm{~K}$ ). The essential results are as follows ${ }^{1}$ :

(a) The decay curves are rather complicated and exhibit several emitting species of different lifetimes. 
The most intense component has a lifetime of $\tau=0.1 \mathrm{~ms}$.

(b) The short lived component with $\tau=0.1 \mathrm{~ms}$ becomes more and more intense on going to shorter emission wavelengths. This is to be expected from the time dependent spectra in Fig. 7 b.

(c) The absolute intensities of the spectra and of the decay curves depend linearly on the excitation intensity. This observation shows that the DF does not originate from triplet-triplet annihilation ${ }^{20}$ *.

By ESR spectroscopy we proved the existence of mobile CT triplet excitons above $100 \mathrm{~K}$ in the crystal biph-TCNB ${ }^{4}$. From the appearance of perfect optical spin polarization and from the measured value of the spin lattice relaxation time $T_{1}$ one obtains the following condition for the lifetime of the triplet excitons: $\tau \ll 5 \mathrm{~ms}$ at $300 \mathrm{~K}^{1,22}$. Therefore the species with a lifetime of $0.1 \mathrm{~ms}$ has to be attributed to the triplet excitons. The very short lifetime of the DF then suggests that the latter is formed via the triplet excitons.

The ESR spetroscopic studies demonstrated that above room temperature the diffusion coefficient, $D_{\mathrm{d}}$, of the triplet excitons is nearly temperature independent ${ }^{4}$. Hence the intensity of $P$-Type DF should also be temperature independent. However, as shown in Fig. $7 \mathrm{a}$ the DF intensity increases strongly with increasing temperature. This supports our conclusion that the DF is of E-Type. We may therefore equate the activation energy $\Delta E_{\mathrm{DF}}=$ $500 \mathrm{~cm}^{-1}$ with the singlet-triplet splitting of the CT-complex ${ }^{20}$.

\section{Characteristic Differences of the Triplet Excitons in the CT-Crystals Anthracene-TCNB and Biphenyl-TCNB}

Table III summarizes some remarkable results concerning the triplet excitons in the crystals anthTCNB and biph-TCNB. The degrees of electron delocalization, $c_{1}{ }^{2}$, given in the first row in Table III were obtained from Equation (3). The activation energies, $E_{\mathrm{D}}$, of the exciton diffusion were determined from the temperature dependence of the ESR line widths.

In the lowest excited biph-TCNB triplet state the contribution of the ionic structure is rather large $\left(c_{1}{ }^{2}=54 \%\right)$, whereas the lowest excited triplet state of anth-TCNB is nearly an unpolar donor triplet state. The striking differences in the properties of the two crystals may be explained on the basis of this difference in the polarity of the lowest excited triplet states.

Let us first consider the different mechanisms leading to DF that were established in Charpter IV. If in the crystal biph-TCNB triplet-triplet annihilation was efficient according to

$$
T_{1}(\mathrm{pol})+T_{1}(\mathrm{pol}) \rightarrow S_{\mathrm{n}}+S_{0}(\text { unpol }),
$$

one of the two polar triplet states $T_{1}(\mathrm{pol})$ would have to be transformed to the unpolar ground state $S_{0}$ (unpol). Such a reaction involving a charge transfer from the donor to the acceptor is rather improbable. However, the reaction

$$
T_{1}(\mathrm{pol})+\text { thermal energy } \rightarrow S_{1}(\mathrm{pol})
$$

\begin{tabular}{|c|c|c|}
\hline & biphenyl-TCNB & anthracene-TCNB \\
\hline CT-character $c_{1}{ }^{2}$ in the triplet state & 0.54 & 0.03 \\
\hline mechanism leading to delayed fluorescence & $\begin{array}{l}\text { thermal transition from } \\
T_{1} \text { to } S_{1} \text { (E-Type) }\end{array}$ & $\begin{array}{l}\text { triplet-triplet } \\
\text { annihilation (P-Type) }\end{array}$ \\
\hline activation energy of triplet exciton diffusion & $E_{\mathrm{D}}=700 \mathrm{~cm}^{-1}$ & $E_{\mathrm{D}}<10 \mathrm{~cm}^{-1}$ \\
\hline $\begin{array}{l}\text { temperature range where mobile triplet excitons were } \\
\text { established }\end{array}$ & $80 \mathrm{~K}<T<350 \mathrm{~K}$ & $4 \mathrm{~K}<T<350 \mathrm{~K}$ \\
\hline dimensionality of the triplet exciton diffusion & one-dimensional & two-dimensional \\
\hline triplet exciton lifetime & $0.1 \mathrm{~ms}$ & $0.5 \mathrm{~ms}$ \\
\hline lifetime of isolated complex & $30 \mathrm{~ms}$ & $10 \mathrm{~ms}$ \\
\hline
\end{tabular}

Table III. The properties of the triplet excitons in the crystals biphenyl-TCNB and anthracene-TCNB.

* Footnote: This is not in contrast to the results of Yagi et al. ${ }^{21}$ who established P.Type delayed CT-fluorescence in biphenyl crystals doped with small amounts of TCNB. In such crystals donor and acceptor assume a configuration different from that in the $1: 1$ crystal biph-TCNB. Ac- cordingly the lowest excited triplet states of CT-crystal and TCNB doped biph crystal should differ drastically in the polarity. This is indicated, for instance, by their different phosphorescence spectra. 
would be favoured in the crystal biph-TCNB ( $E$ Type), as the thermal transition from the polar triplet state to the lowest excited CT singlet state $S_{1}$ (pol) requires only a small change in the charge transfer. In addition the energy difference between the states $T_{1}$ and $S_{1}$ is most probably very small $\left(<2000 \mathrm{~cm}^{-1}\right)^{*}$.

In the crystal anth-TCNB the lowest excited triplet state $T_{1}$ is rather unpolar and much lower in energy than the lowest excited singlet state $S_{1}(\mathrm{pol})$. Thus the thermal transition to the $S_{1}$-state is not favoured. On the other hand there exist unpolar higher excited singlet states $S_{\mathrm{n}}$ (unpolar) fullfilling the energy condition $E\left(S_{\mathrm{n}}\right)<2 E\left(T_{1}\right)$. In this case the annihilation reaction $T_{1}$ (unpol) $+T_{1}$ (unpol) $\rightarrow S_{0}$ (unpol) $+S_{\mathrm{n}}$ (unpol) would be possible.

Comparison of the results in row 3 and 4 of Table III shows that the hopping process of a polar charge transfer triplet state requires a thermal activation. Of considerable importance is the question about the physical significance of the activation energy $E_{\mathrm{D}}$.

One possible interpretation of $E_{\mathrm{D}}$ is that polar triplet excitons are self trapped by polarization of their environment. The polarization energy $P$ of an exciton with charge transfer character $c_{1}{ }^{2}$, that is embedded in a CT-crystal, may be calculated by a point-charge approximation if the polarizabilities of the donor and the acceptor are known ${ }^{1}$. In this approximation $P$ is proportional to $c_{1}{ }^{4}$ and one estimates $P=2000 \mathrm{~cm}^{-1}$ for biph-TCNB $\left(c_{1}{ }^{2}=0.54\right)$ and $P=5 \mathrm{~cm}^{-1}$ for anth-TCNB $\left(c_{1}{ }^{2}=0.03\right)$. For both crystals these polarization energies are of the same order of magnitude as the corresponding activation energies $E_{\mathrm{D}}$. This suggests that the polarization of the crystal by the excited CT-state contributes considerably to the exciton self-trapping.

Another origin of the self trapping of CT triplet excitons may be a distortion of the lattice upon excitation ${ }^{24}$. Such a distortion is expected if the

* Footnote: In solution E-Type delayed fluorescence of CTcomplexes has already been found by Beens and Weller ${ }^{23}$.

1 H. Möhwald, Thesis, Universität Göttingen 1974.

2 D. Allender, J. Bray, and J. Bardeen, Phys. Rev. B 7, 1020 [1973].

${ }^{3}$ E. B. Yagubskii and M. L. Khidekel, Russ. Cem. Rev. 41 (12), 1011 [1972].

${ }^{4}$ H. Möhwald and E. Sackmann, Chem. Phys. Letters 21, 43 [1973].

5 H. Möhwald and E. Sackmann, Solid State Comm. (1974), in press.

${ }^{6}$ B. Nickel and U. Nickel, Ber. Bunsenges. 76, 584 [1972]. equilibrium configurations in the excited state and in the ground state of the complex are different ${ }^{25}$. A simple distortion with a strong effect on the triplet energy would be a decrease in the interplanar distance. The decrease in the distance of a donoracceptor pair leads to an increase in the Coulomb energy and thus lowers the triplet energy. In this case an exciton can only jump to an adjacent site if the trapped triplet state comes into resonance with the triplet exciton band of the unperturbed lattice. Thus the exciton motion demands a strong excitonphonon coupling ${ }^{26}$. According to Briegleb ${ }^{16}$ a change in $d$ by $\Delta d=0.01 \AA$ leads to a change in the Coulomb energy by $\Delta C=100 \mathrm{~cm}^{-1}$ for a charge transfer complex of $c_{1}{ }^{2}=1$. In biph-TCNB $\left(c_{1}{ }^{2}=\right.$ $0.54)$ a decrease in $d$ of $\Delta d=0.13 \AA$ would be necessary if the corresponding increase in the Coulomb energy should account for the total experimental activation energy $E_{\mathrm{D}}=700 \mathrm{~cm}^{-1}$. Most probably both mechanism are important for the self trapping process.

The triplet excitons differ also in the dimensionality of the diffusion. The triplet excitons in biph-TCNB $\left(c_{1}{ }^{2}=0.54\right)$ move preferentially along the stack axis (one-dimensional excitons). In the crystal anth-TCNB $\left(c_{1}{ }^{2}=0.03\right)$, however, the triplet exciton motion along the stack axis would have to proceed via the lowest excited acceptor triplet state. This state presents a very high barrier of about $8000 \mathrm{~cm}^{-1}$. Since all donor molecules are positioned on the (001)-planes the two-dimensional exciton motion within these planes is favoured.

\section{Acknowledgement}

We thank Prof. Dr. A. Weller for most helpful and critical discussions. We are greatly indebted to Dr. B. Nickel for help with the optical experiments and their interpretation. The generous help and advice of Dr. W. Kühnle with the purification of the substances is gratefully acknowledged.

\footnotetext{
7 S. Kumakura, F. Iwasaki, and Y. Saito, Bull. Chem. Soc. Jap. 40, 1826 [1967].

8 Ph. Kottis and R. Lefèbvre, J. Chem. Phys. 34, 379 [1964].

9 J. N. Murrell, Theory of the Electronic Spectra of Organic Molecules, Methuen Ltd., London 1963, pp. $279 \mathrm{ff}$.

10 H. Hayashi, S. Iwata, and S. Nagakura, J. Chem. Phys. 50, 993 [1969].

11 H. Beens, J. de Jong, and A. Weller, Colloque Ampère 15, 289 [1969].

12 P. Krebs, Thesis, Universität Stuttgart 1973.

13 H. Möhwald and E. Sackmann, Chem. Phys. Letters, in press.
} 
14 S. P. McGlynn, T. N. Misra, and E. F. McCoy, International Symposium on Luminescence, Karl-Thiemig-Verlag 1966, Munich (Germany) 1965.

15 J. de Jong, Thesis, Free University, Amsterdam 1970.

16 G. Briegleb, Elektronen-Donator-Acceptor Komplexe, Springer-Verlag, Berlin 1961.

$17 \mathrm{H}$. Beens and A. Weller, Chem. Phys. Letters 2, 140 [1968].

18 D. Haarer and N. Karl, Chem. Phys. Letters 21, 49 [1973].

19 S. P. McGlynn, J. D. Boggus, and E. Elder, J. Chem. Phys. 32, 357 [1960].

20 C. A. Parker, Photoluminescence of Solutions, Elsevier, Amsterdam 1968.

21 M. Yagi, S. Nagakura, and H. Hayashi, Chem. Phys. Letters 18, 272 [1973].

22 D. Haarer and H. C. Wolf, Phys. Stat. Sol. 23, K 117 [1969].
${ }^{23} \mathrm{H}$. Beens and A. Weller, in "Molecular Luminescence", E. C. Lim, New York 1969.

$24 \mathrm{~K}$. Cho and Y. Toyozawa, J. Phys. Soc. Japan 30, 1555 [1971].

25 R. M. Hochstrasser, S. K. Lower, and C. Reid, J. Chem. Phys. 41, 1073 [1964].

26 J. A. Sussmann, Phys. kondens. Materie 2, 146 [1964].

27 P. Krebs, E. Sackmann, and J. Schwarz, Chem. Phys. Letters 8, 417 [1971].

28 D. Haarer and H. C. Wolf, Mol. Cryst. Liqu. Cryst. 10, 359 [1970].

29 H. Tsuchiya, F. Marumo, and Y. Saito, Acta Cryst. B 28, 1935 [1972].

30 H. P. Müller, P. Thoma, and G. Vaubel, Phys. Stat. Sol. 23, K 117 [1969]. 